ARTICLE

DOI: $10.1038 / \mathrm{s} 41467-018-04028-8$

\title{
Molecular doping enabled scalable blading of efficient hole-transport-layer-free perovskite solar cells
}

Wu-Qiang Wu 10 1,2, Qi Wang 1,2, Yanjun Fang², Yuchuan Shao1,2, Shi Tang2, Yehao Deng ${ }^{1,2}$, Haidong Lu Ye Liu' ${ }^{2}$, Tao Li ${ }^{3}$, Zhibin Yang ${ }^{1}$, Alexei Gruverman ${ }^{3} \&$ Jinsong Huang ${ }^{1,2}$

The efficiencies of perovskite solar cells (PSCs) are now reaching such consistently high levels that scalable manufacturing at low cost is becoming critical. However, this remains challenging due to the expensive hole-transporting materials usually employed, and difficulties associated with the scalable deposition of other functional layers. By simplifying the device architecture, hole-transport-layer-free PSCs with improved photovoltaic performance are fabricated via a scalable doctor-blading process. Molecular doping of halide perovskite films improved the conductivity of the films and their electronic contact with the conductive substrate, resulting in a reduced series resistance. It facilitates the extraction of photoexcited holes from perovskite directly to the conductive substrate. The bladed hole-transport-layerfree PSCs showed a stabilized power conversion efficiency above $20.0 \%$. This work represents a significant step towards the scalable, cost-effective manufacturing of PSCs with both high performance and simple fabrication processes.

\footnotetext{
${ }^{1}$ Department of Applied Physical Sciences, University of North Carolina, Chapel Hill, NC 27599, USA. ${ }^{2}$ Department of Mechanical and Materials Engineering, University of Nebraska-Lincoln, Lincoln, NE 68588, USA. ${ }^{3}$ Department of Physics and Astronomy, University of Nebraska-Lincoln, Lincoln, NE 68588, USA. Correspondence and requests for materials should be addressed to J.H. (email: jhuang@unc.edu)
} 
S ince their first report in 2009, halide perovskite solar cells (PSCs) have attracted enormous interest as emerging lightto-electricity conversion optoelectronic devices with both high efficiency and ease of solution processability ${ }^{1-3}$. To date, the power conversion efficiencies (PCEs) of solution-processed PSCs have been certified at well above $20 \%^{4-6}$. However, all topperforming PSCs were fabricated by a spin-coating method which is unsuitable for high throughput and scalable module production. Several scalable film deposition techniques have been developed for PSC fabrication, such as doctor-blading ${ }^{7-10}$, spray deposition $^{11}$, slot-die coating ${ }^{2}$, solution printing ${ }^{13}$, and electrodeposition ${ }^{14}$. In particular, doctor-blade coating with a controlled substrate temperature has gained most success in depositing highquality perovskite films with large grains and controllable optoelectronic properties ${ }^{15}$. Moreover, blade coating has great potential to be scaled up for roll-to-roll fabrication. Encouragingly, the PCEs of PSCs with bladed perovskite layers have steadily increased to above 19\%, approaching the levels of their spin-coated counterparts ${ }^{13,16}$.

A next step towards the scalable fabrication of PSCs is to coat the charge transport layers by scalable processes. However, the existing multiple-layer device structures, either $\mathrm{p}-\mathrm{i}-\mathrm{n}$ or $\mathrm{n}-\mathrm{i}-\mathrm{p}$ structures with a hole transport layer (HTL), perovskite, and electron transport layer (ETL), impose a great challenge on the scalable fabrication of PSCs in a cost-effective manner ${ }^{17-20}$. First, high-performance organic hole-transporting materials, such as 2,2',7,7'-Tetrakis[N,N-di(4-methoxyphenyl)amino]9,9'-spirobifluorene (spiro-OMeTAD), are much more expensive than perovskite materials. Second, for the devices with $\mathrm{p}-\mathrm{i}-\mathrm{n}$ structure, it is difficult to blade-coat perovskite films onto a hydrophobic HTL, e.g., poly(bis(4-phenyl)(2,4,6-trimethylphenyl)amine) (PTAA), while a hydrophilic HTL, e.g., $\mathrm{NiO}_{x}$, is difficult to form on plastic substrates at low temperature, nor can easily be coated at optimal thickness and electronic quality to achieve the highest device efficiency. Hence, we believe that the elimination of the HTL from the PSC device architecture can be an effective way to solve this challenge. By reducing one step of the standard coating process, at least one third of the manufacture cost and time can be saved, in addition to savings of material cost for the HTL.

The reported ambipolar charge transport characteristics of halide perovskite materials endow them with great potential to construct PSCs with simplified device architecture, such as HTLfree PSCs with either conventional or inverted structure ${ }^{21-23}$. Yet, a critical challenge for HTL-free PSCs is the ineffective hole collection by indium tin oxide (ITO) because of mismatched work functions between ITO and the perovskite, which generally causes significant efficiency $\operatorname{loss}^{23-25}$. In addition, the construction of HTL-free devices by the blade-coating method faces more challenges due to rougher and more non-uniform perovskite films, than do those prepared by spin-coating with the antisolvent method.

Here we report a molecular-doping strategy for a perovskite layer to doctor-blade HTL-free PSCs with a simplified device structure of ITO/perovskite/ETL/Cu. Doping methylammonium lead iodide $\left(\mathrm{MAPbI}_{3}\right)$ films with 2,3,5,6-tetrafluoro-7,7,8,8-tetracyanoquinodimethane (F4TCNQ), a strong electron-withdrawing molecule, leads to a modified ITO/MAPbI ${ }_{3}$ interface with favorable band bending, which facilitates the extraction of photoexcited holes from perovskite to ITO electrode. Combining additive engineering and solvent annealing, high-quality $\mathrm{MAPbI}_{3}$ films are obtained with full surface coverage, micrometer-sized grains, and improved crystallinity. By employing a bladed and doped $\mathrm{MAPbI}_{3}$ film as the photoactive layer in a HTL-free device structure, a stabilized PCE over $20.0 \%$ with almost no $J-V$ hysteresis is obtained.

\section{Results}

Perovskite films fabrication and molecular doping. The $\mathrm{MAPbI}_{3}$ perovskite films were directly deposited onto blank ITO glass substrates by a previously reported doctor-blading method ${ }^{9}$, which is schematically illustrated in Fig. 1a. Before blade coating, the ITO substrates were cleaned by a UV-ozone treatment to improve their wettability to perovskite solution ${ }^{23}$. $\mathrm{MAPbI}_{3}$ precursor dissolved in N,N-diethylformamide (DMF) containing $\mathrm{p}$ type dopant (F4TCNQ) was dripped onto substrates which were heated at $150^{\circ} \mathrm{C}$, followed by a quick blading process to spread the solution onto the substrates. After blading, the perovskite precursor solution dried in a few seconds, and the substrates were quickly removed from the hot plate and annealed at $100^{\circ} \mathrm{C}$ for varied durations. The bladed perovskite films showed thicknesses of $500 \pm 20 \mathrm{~nm}$ from cross-sectional imaging (Fig. 1b) by scanning electron microscopy (SEM). To implement molecular doping of the perovskite layer, F4TCNQ with chemical structure shown in Fig. 1a was dissolved in DMF separately, and then added to the as-prepared $\mathrm{MAPbI}_{3}$ precursor solution. Perovskite films made without or with F4TCNQ were denoted as $\mathrm{MAPbI}_{3}$ or $\mathrm{MAPbI}_{3}: \mathrm{F} 4 \mathrm{TCNQ}$, respectively.

The doping of perovskite is expected to change the work function of the films. Kelvin probe force microscopy (KPFM) was used to determine the surface potential change of perovskite films upon F4TCNQ mixing. In addition to direct mixing of F4TCNQ and $\mathrm{MAPbI}_{3}$ in precursor solution, $\mathrm{MAPbI}_{3}: \mathrm{F} 4 \mathrm{TCNQ}$ was prepared by solid diffusion via heating F4TCNQ powder on $\mathrm{MAPbI}_{3}$ and then removing the excess F4TCNQ. The topography and contact potential difference (CPD) images of pristine $\mathrm{MAPbI}_{3}$ and $\mathrm{MAPbI}_{3}: \mathrm{F} 4 \mathrm{TCNQ}$ films by either standard solution mixing or solid diffusion are shown in Fig. 1c-h. For better comparison, the three CPD images are flattened and presented in the same color scale, while the histograms of the absolute CPD values of the three types of films are plotted in Fig. 1i. The pure $\mathrm{MAPbI}_{3}$ film has coarse grains and uniform CPD distribution (Fig. 1c, f). In contrast, solution mixed $\mathrm{MAPbI}_{3}$ :F4TCNQ film, in which uniform doping is expected, has fine grains and greater heterogeneity of the CPD distribution (Fig. 1d, g). For the sample with solid-diffused F4TCNQ, some small F4TCNQ particles were placed on top of a $\mathrm{MAPbI}_{3}$ film, and the CPD imaging was conducted around the edge (the green color line as indicated in Fig. 1h) to include both neat and doped perovskite regions. Such method leads to a clear edge between diffused and undiffused regions that can be visualized in the CPD image (Fig. 1h), thus the CPD heterogeneity is even higher. The change of absolute CPD caused by F4TCNQ doping is clearly demonstrated in Fig. 1i. A decrease of surface potential or in other words an increase of work function was observed for the $\mathrm{MAPbI}_{3}$ :F4TCNQ films, irrespective of direct mixing or solid diffusion. The increase of heterogeneity for the $\mathrm{MAPbI}_{3}$ :F4TCNQ films is also reflected in the broadening of the curves. This confirmed p-type doping of $\mathrm{MAPbI}_{3}$ by F4TCNQ. Specifically, after the addition of electronwithdrawing F4TCNQ molecule to the $\mathrm{MAPbI}_{3}$, owing to the deep lowest unoccupied molecular orbital (LUMO) level of F4TCNQ, electrons in the valance band of $\mathrm{MAPbI}_{3}$ can be thermally activated into the LUMO level of F4TCNQ, which leaves holes in $\mathrm{MAPbI}_{3}$ and thus causes its p-doping (Fig. 1j). As a result, the hole concentration in the $\mathrm{MAPbI}_{3}$ film increased. The carrier concentration change upon F4TCNQ doping has been evaluated by analyzing the dark capacitance vs. voltage $(C-V)$ characteristics of the perovskite devices (Supplementary Fig. 1). Charge density profiles were extracted from the $C-V$ curves using Mott-Schottky analysis ${ }^{26,27}$. The carrier concentration in the perovskite layer was calculated to be $0.6 \times 10^{16} \mathrm{~cm}^{-3}$ in the neat $\mathrm{MAPbI}_{3}$ film which increased to $2.4 \times 10^{16} \mathrm{~cm}^{-3}$ after the addition of $0.03 \mathrm{wt} \%$ F4TCNQ. This molecular p-type doping 

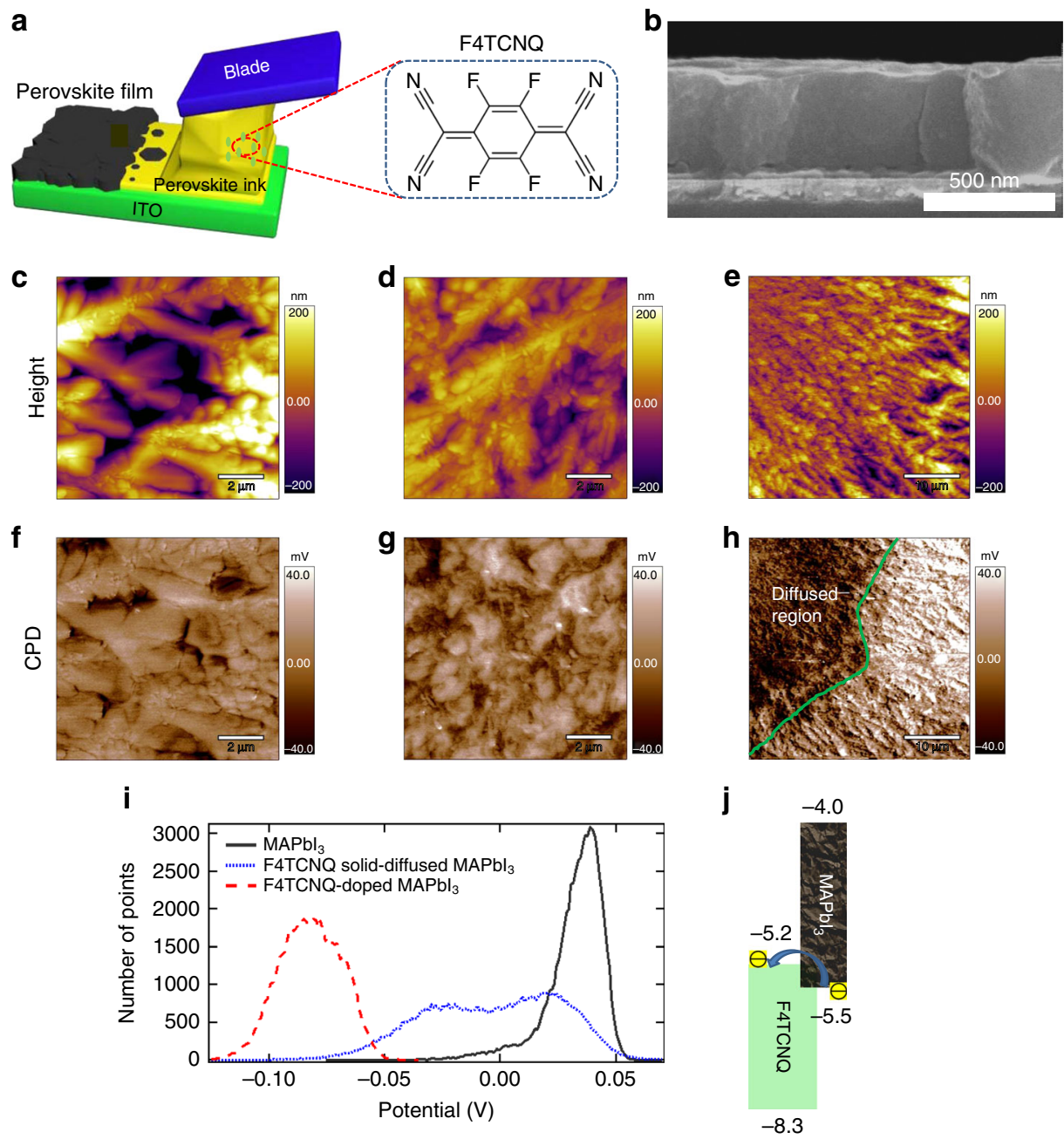

Fig. 1 Doctor blading and doping of perovskite films by F4TCNQ. a Schematic illustration of a doctor-bladed perovskite film and the chemical structure of F4TCNQ dopant. $\mathbf{b}$ Cross-sectional SEM image of the $\mathrm{MAPb}_{3}$ film deposited directly onto ITO glass via bladed coating at $150{ }^{\circ} \mathrm{C}$, showing the film thickness of around $500 \mathrm{~nm}$. Topography KPFM images of $\mathbf{c}, \mathbf{f} \mathrm{MAPbl}_{3}, \mathbf{d}, \mathbf{g}$ F4TCNQ-doped MAPbl ${ }_{3}$, and $\mathbf{e}$, $\mathbf{h}$ F4TCNQ solid-diffused MAPbl 3 films. CPD represents the contact potential difference between the tip and sample's surface. $\mathbf{i}$ Surface potential profiles of different perovskite films as indicated. $\mathbf{j}$ Schematic illustration of the energy diagram and electron transfer process for MAPbl 3 :F4TCNQ blends

strategy enabled the fabrication of an HTL-free PSC with a simplified device configuration but still effective $\mathrm{p}-\mathrm{n}$ heterojunction (i.e., p-doped perovskite and n-type ETL), and thus enhanced device performance.

Electronic properties of doped perovskite films. The electronic properties of the $\mathrm{MAPbI}_{3}: \mathrm{F} 4 \mathrm{TCNQ}$ films were investigated by comparing their current density-voltage $(J-V)$ characteristics with pristine $\mathrm{MAPbI}_{3}$ films deposited on glass substrates in the dark condition. The lateral conductivity was measured across gold electrodes spaced $100 \mu \mathrm{m}$ apart (Fig. 2a). As shown in Fig. 2b, the $\mathrm{MAPbI}_{3}: \mathrm{F} 4 \mathrm{TCNQ}$ film exhibited a nearly tenfold increase in conductivity over the neat $\mathrm{MAPbI}_{3}$ film, which confirmed that F4TCNQ doped $\mathrm{MAPbI}_{3}$. The charge transfer from $\mathrm{MAPbI}_{3}$ to F4TCNQ is expected to cause quenching of photoluminescence $(\mathrm{PL})$ from the $\mathrm{MAPbI}_{3}$ layer, which was measured by using time-resolved photoluminescence (TRPL) decay for $\mathrm{MAPbI}_{3}$ films deposited on glass substrates (Fig. 2c). The $\mathrm{MAPbI}_{3}$ :F4TCNQ prepared on glass substrate showed a PL lifetime of $65 \mathrm{~ns}$, which is fourfold shorter than the PL lifetime of pristine $\mathrm{MAPbI}_{3}$ film (268 ns) on glass.

The relative large molecular size of F4TCNQ likely prevents its integration into the lattice of $\mathrm{MAPbI}_{3}$. Therefore, we speculate that F4TCNQ molecules most probably remain at the grain boundary (GB) regions of perovskite films and/or perovskite/ electrode interfaces. To determine the lateral distribution of F4TCNQ molecules, the local dark conductivity at grains and GBs was measured using conducting atomic force microscopy (cAFM). Figure $2 \mathrm{~d}$ illustrates the c-AFM setup, where the microscope tip was positioned at locations randomly chosen from topographic AFM images (Fig. 2e, g). The tip bias was scanned in the range +2.0 to $-2.0 \mathrm{~V}$ at a rate of $0.14 \mathrm{~V} \mathrm{~s}^{-1}$. For the neat $\mathrm{MAPbI}_{3}$ film, the dark currents were similar at the grain and GB regions (Fig. $2 \mathrm{f}$ and Supplementary Fig. 2a, b), which is in accordance with previous reporting ${ }^{28}$. In sharp contrast, the dark currents measured at the GB regions of the $\mathrm{MAPbI}_{3}: \mathrm{F} 4 \mathrm{TCNQ}$ film were notably higher (Fig. $2 \mathrm{~h}$ and Supplementary Figs. $2 \mathrm{c}, \mathrm{d}$ ), elucidating the remarkably improved conductivity at the GB region after molecular doping of $\mathrm{MAPbI}_{3}$ by F4TCNQ. At the regions close to GBs, the F4TCNQ molecules and $\mathrm{MAPbI}_{3}$ undergo charge transfer, leading to an increase in carrier density in $\mathrm{MAPbI}_{3}$ and thus the enhanced dark conductivity ${ }^{29}$.

Morphologies and crystallinities of perovskite films. Highquality perovskite films with large grains, high crystallinity and complete surface coverage are also crucial to achieving high- 
a

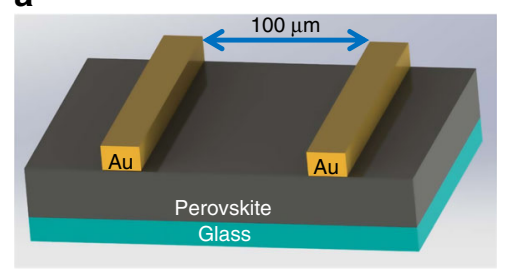

C

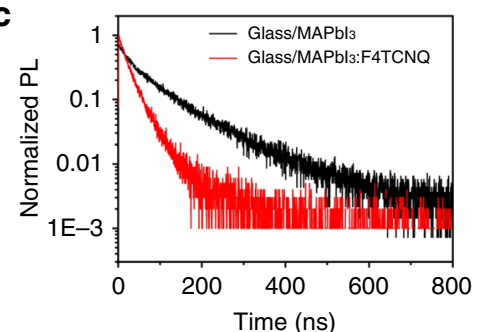

e

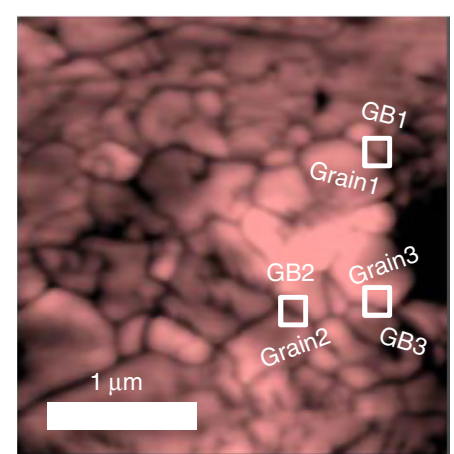

g

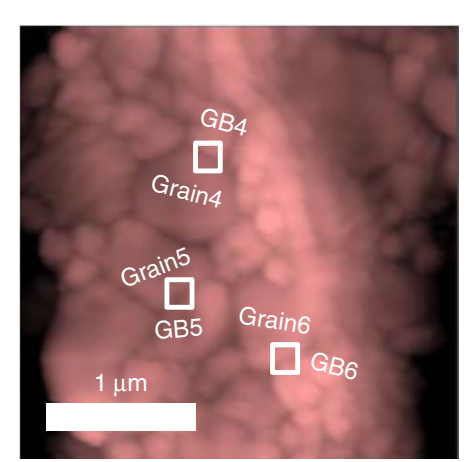

b

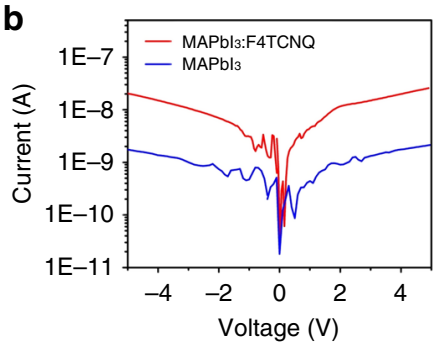

d

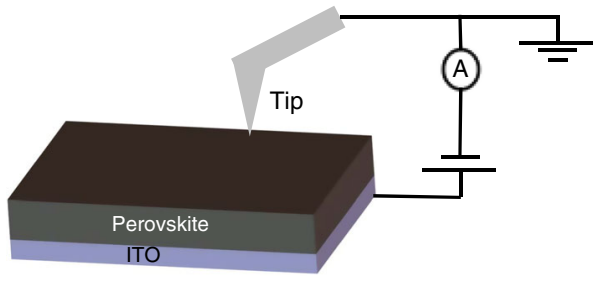

f
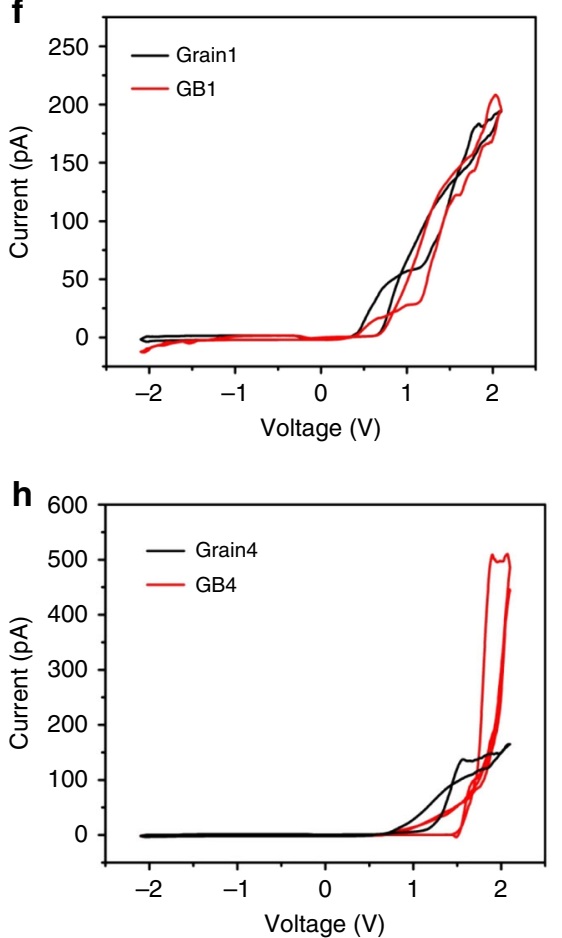

Fig. 2 Conductivities and photoluminescence lifetimes of neat or doped perovskite films. a Geometry for the lateral conductivity measurement: the perovskite films were $500 \mathrm{~nm}$ thick, with $1 \mathrm{~mm}$ gold (Au) electrodes separated by $100 \mu \mathrm{m}$. $\mathbf{b} \mathrm{J}-\mathrm{V}$ curves of neat or F4TCNQ-doped MAPbl ${ }_{3}$ deposited on normal glass substrates, where the current was measured along the lateral direction. $\mathbf{c}$ TRPL decay curves of glass/MAPbl ${ }_{3}$ and glass/MAPbl 3 :F4TCNQ. PL lifetimes were calculated by single exponential fitting. $\mathbf{d}$ c-AFM setup. Topographic AFM images of $\mathbf{e} \mathrm{MAPbl}_{3}$ and $\mathbf{g}$ MAPbl $\mathrm{I}_{3}$ :F4TCNQ films, with locations where the c-AFM tip measured the grain and GB currents. Local dark currents measured at the GBs and on the grains for the $\mathbf{f} \mathrm{MAPbl}_{3}$ film and $\mathbf{h} \mathrm{MAPb}{ }_{3}$ : F4TCNQ film

performance HTL-free devices. The blending of F4TCNQ in the perovskite in a small percentage (ranged from 0.01 to $0.05 \mathrm{wt} \%$ ) did not notably change the morphology and grain size of the perovskite films, as shown by the SEM images in Supplementary Fig. 3, nor cause a drastic change of crystallinity, as shown by the XRD patterns of bladed $\mathrm{MAPbI}_{3}$ films prepared with different amounts of F4TCNQ in Supplementary Fig. 4. Accordingly, methylammonium hypophosphite (MHP) and methylammonium chloride $(\mathrm{MACl})$ were introduced as additives to the precursor solution, and co-solvents (dimethyl sulfoxide (DMSO)/ chlorobenzene (CBZ)) post-annealing process was also combined to achieve a high-quality and high-crystalline $\mathrm{MAPbI}_{3}$ film
(Supplementary Figs. 5, 6 and 7 for SEM images and XRD patterns of bladed $\mathrm{MAPbI}_{3}$ films prepared with different amounts of MHP as well as using co-solvents annealing technique in the absence or presence of $\mathrm{MACl})^{9,10,16,30,31}$. Figure $3 \mathrm{a}, \mathrm{b}$ show representative SEM images of the bladed perovskite films after optimization, which featured a uniform morphology that extended hundreds of micrometers. The perovskite domains were densely packed together, and without observable voids at domain boundaries (Fig. 3a). It should be noted that these domains are not single crystalline grains. Each micrometer-scale convection domain was comprised of micro-sized grains ranging from 800 to $1700 \mathrm{~nm}$ (average above $1 \mu \mathrm{m}$, Fig. 3b). 

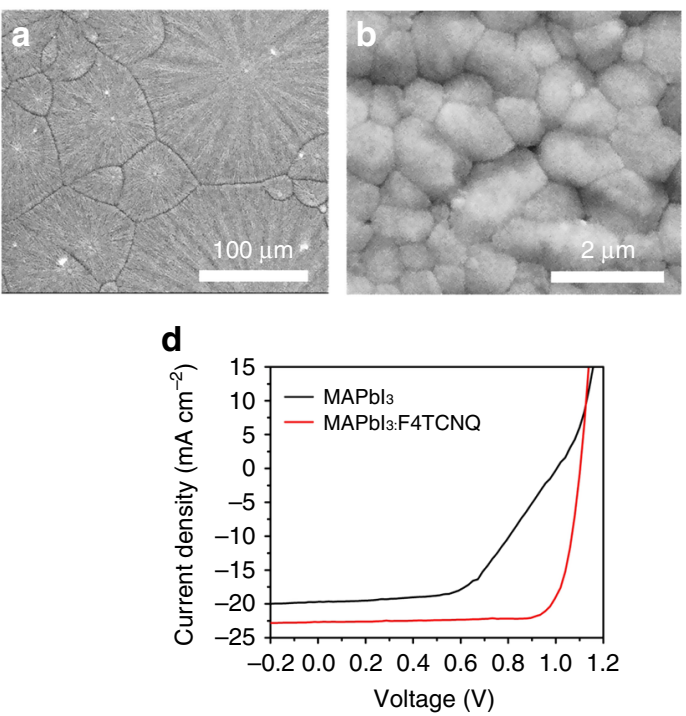

f

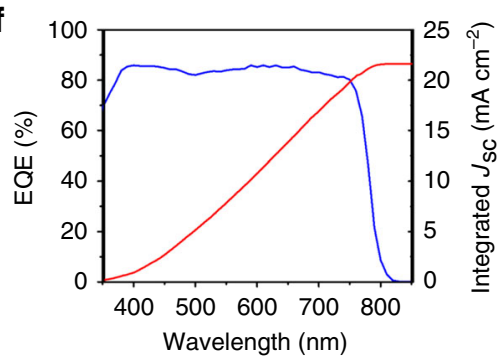

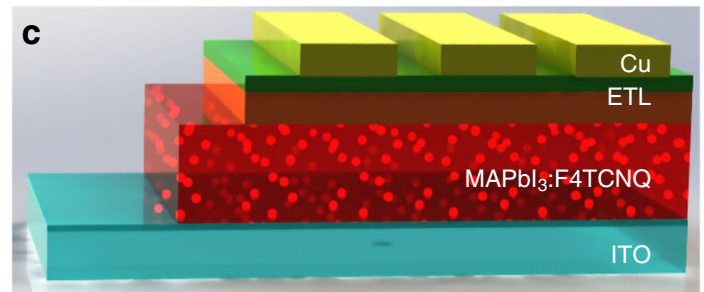

e
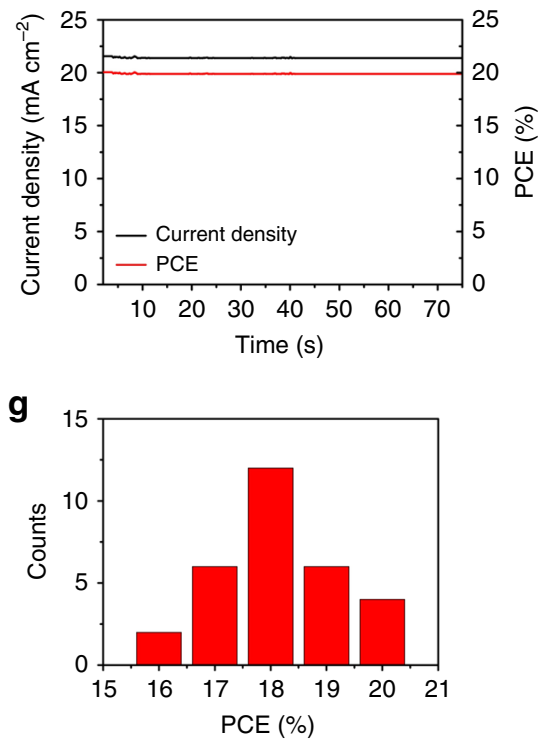

Fig. 3 Perovskite film morphology, device structure, and photovoltaic performance. a Low magnification and $\mathbf{b}$ high magnification SEM images of bladed and doped $\mathrm{MAPbl}_{3}$ film prepared with $\mathrm{MHP}(0.225$ wt\%) and $\mathrm{MACl}(0.5 \mathrm{wt} \%)$ as additive, followed by co-solvents annealing treatment. c Schematic illustration of the HTL-free device configuration. d J-V characteristics, e Steady-state current and stabilized PCE measured at a maximum power point (0.93 V), f EQE and integrated current density, and $\mathbf{g}$ PCE histogram of PSCs based on MAPbl 3 :F4TCNQ films (with 0.03 wt\% F4TCNQ)

\begin{tabular}{|c|c|c|c|c|c|}
\hline PSCs & $J_{s c}\left(m A c m^{-2}\right)$ & $V_{\mathrm{oc}}(\mathrm{mV})$ & $\eta(\%)$ & $\begin{array}{l}\text { Average } \\
\eta(\%)\end{array}$ & $\mathbf{F F}$ \\
\hline $\mathrm{MAPbl}_{3}$ & 19.7 & 1.00 & 11.0 & $9.52 \pm 0.72$ & 0.56 \\
\hline $\begin{array}{l}\text { MAPbl }_{3}: \\
\text { F4TCNQ }\end{array}$ & 22.7 & 1.10 & 20.2 & $18.85 \pm 0.35$ & 0.81 \\
\hline
\end{tabular}

Data for average PCE $(\eta)$ were calculated from at least 30 devices

Photovoltaic performances of doped perovskite devices. The impact of molecular doping by F4TCNQ on photovoltaic performance was investigated in detail. As illustrated in Fig. 3c, inverted HTL-free PSCs were fabricated with a device configuration of $\mathrm{ITO} / \mathrm{MAPbI}_{3}: \mathrm{F} 4 \mathrm{TCNQ} / \mathrm{ETL} / \mathrm{Cu}$. The corresponding photovoltaic performances for PSCs based on different F4TCNQ concentrations have been displayed in the Supplementary Table 1, which indicated the optimal F4TCNQ concentration was $0.03 \mathrm{wt} \%$. Figure $3 \mathrm{~d}$ shows the current density vs. voltage $(J-V)$ characteristics of PSCs based on the pristine $\mathrm{MAPbI}_{3}$ films and $\mathrm{MAPbI}_{3}$ :F4TCNQ films (with $0.03 \mathrm{wt} \%$ F4TCNQ), with photovoltaic parameters summarized in Table 1 . The reference PSC without F4TCNQ (i.e., $\mathrm{MAPbI}_{3}$ ) exhibited a poor PCE of just $11.0 \%$, which is in accordance with that of most reported HTL-free PSCs ${ }^{23,24}$. Surprisingly, with the addition of F4TCNQ, notable concurrent enhancements of the $J_{\mathrm{sc}}, V_{\mathrm{oc}}$, and FF have been achieved. The champion cell based on $\mathrm{MAPbI}_{3}: \mathrm{F} 4 \mathrm{TCNQ}$ film achieved the highest PCE of $20.2 \%$, with a $J_{\mathrm{sc}}$ of $22.7 \mathrm{~mA} \mathrm{~cm}^{-2}$, a $V_{\mathrm{oc}}$ of $1.10 \mathrm{~V}$, and a FF of 0.81 . To the best of our knowledge, this is the record efficiency for the bladed PSCs in the absence of HTLs. For comparison, the PSC based on the PTAA HTL and undoped $\mathrm{MAPbI}_{3}$ layer has been fabricated, which showcases a PCE of $19.5 \%$, along with a $J_{\mathrm{sc}}$ of $22.1 \mathrm{~mA} \mathrm{~cm}^{-2}$, a $V_{\text {oc }}$ of $1.12 \mathrm{~V}$, and a FF of 0.79 . This result is in good agreement with our previous reports $8,9,16$. It is worth noting that the demonstrated HTL-free device showed similar or even better photovoltaic performance, highlighting the effectiveness of such a doping strategy for constructing high-performance PSCs with not only a simpler structure, but also less deposition steps and lower material cost. The $J-V$ curves of $\mathrm{MAPbI}_{3}: \mathrm{F} 4 \mathrm{TCNQ}$-based device were measured in both reverse and forward scan modes at a scan rate of $0.1 \mathrm{~V} \mathrm{~s}^{-1}$, and almost no hysteresis was observed (Supplementary Fig. 8). Figure $3 \mathrm{e}$ shows the stabilized photocurrent density of this device as measured at $0.93 \mathrm{~V}$, giving a stabilized PCE exceeding 20.0\% under continuous light soaking. This high stabilized PCE output is among the best record for HTL-free PSCs, and is quite remarkable given that the device is prepared using a scalable fabrication method. Figure $3 \mathrm{f}$ shows the external quantum efficiency (EQE) of the champion device. The integrated $J_{\mathrm{sc}}$ from the EQE measurement agreed well with the $J_{\mathrm{sc}}$ values measured under AM 1.5 G one sun illumination (Fig. 3d). The fabrication procedure was repeated to confirm the reliability and reproducibility of the results. The PCE histogram was collected from 30 independent cells (Fig. 3g), revealing that more than $73 \%$ of the cells had PCEs above $18.0 \%$, and more than $93 \%$ had PCEs higher than $17.0 \%$ under one sun illumination. A study of the stability for the devices with or without HTL has been conducted. 
The devices were stored under ambient conditions (where humidity and temperature were about $20 \%$ and $25^{\circ} \mathrm{C}$, respectively) without any encapsulation. As compared to the devices with HTL, the HTL-free device exhibits a slightly better stability, which maintained over $92 \%$ of its initial PCE after storage for 500 h (Supplementary Fig. 9).

It is noted from Fig. $3 \mathrm{~d}$ and Table 1 that the almost twofold efficiency enhancement in the F4TCNQ-doped devices primarily originates from the remarkable FF increase. Further analysis of the $J-V$ curves in Fig. $3 \mathrm{~d}$ revealed that F4TCNQ doping of the perovskite layer significantly reduced device series resistance $\left(R_{\mathrm{s}}\right)$ from $25.6 \Omega \mathrm{cm}^{2}$ for undoped device to $2.6 \Omega \mathrm{cm}^{2}$ for doped device. The influence of $R_{\mathrm{s}}$ on device FF has been well established in diode based solar cells ${ }^{32}$, according to the classical equation based on diode equation:

$$
I=I_{\mathrm{L}}-I_{0} \exp \left[\frac{q\left(V+I R_{\mathrm{s}}\right)}{n k T}\right]
$$

where $I$ is the device output current, $I_{\mathrm{L}}$ is the maximum light generated current, $I_{0}$ is saturated dark current, $R_{\mathrm{s}}$ is series resistance, $n$ is ideal factor, $k$ is Boltzmann constant, $T$ is temperature. Assuming a diode ideal factor of 1 , the FFs for the undoped and doped devices were calculated to be $53 \%$ and $83 \%$ from Eq. (1), which are very close to measured values of $56 \%$ and $81 \%$, respectively. This indicates the increased FF for the devices with doped $\mathrm{MAPbI}_{3}$ is mainly caused by the reduction of the series resistance. To find out the origin of reduced series resistance by F4TCNQ doping, energy level diagrams are constructed and shown in Fig. 4 for the perovskite/ITO interface with or without F4TCNQ doping. Here the energy levels were extracted from the literature for the films made with same composition and similar method ${ }^{33,34}$. For undoped $\mathrm{MAPbI}_{3}$, the Fermi level of pristine $\mathrm{MAPbI}_{3}$ (nearly intrinsic) is slightly lower than that of ITO $(-4.7 \mathrm{eV})^{34}$. The Fermi level lines up during contact formation and causes a downward band-bending in $\mathrm{MAPbI}_{3}$, which forms a barrier for hole extraction from $\mathrm{MAPbI}_{3}$ to ITO (Fig. 4a). This would also increase the possibility of charge recombination and increase series resistance at the ITO/ perovskite interface. In contrast, the incorporation of F4TCNQ in the $\mathrm{MAPbI}_{3}$ films turns the $\mathrm{MAPbI}_{3}$ to more p-type, particularly at the interface of ITO and $\mathrm{MAPbI}_{3}$, which should avert band-bending direction (Fig. 4b). This was confirmed by surface potential measurement that the F4TCNQ doping can increase work function of perovskite by $\sim 0.1$ to $0.2 \mathrm{eV}$ (Fig. 1i). As is shown in Fig. 4b, the F4TCNQ doping results in an upward band bending at the ITO/MAPbI 3 :F4TCNQ interface. In this case, the hole transfer from F4TCNQ-doped $\mathrm{MAPbI}_{3}$ to
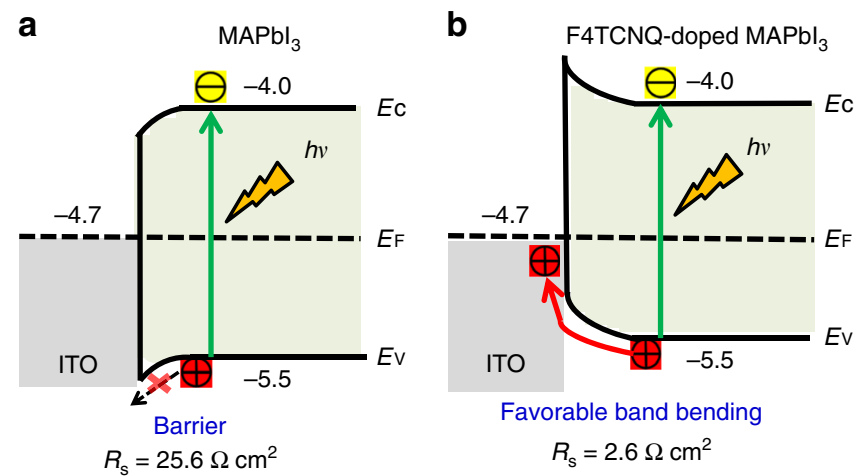

Fig. 4 Interfacial hole transfer dynamics. Schematic illustrations of hole transfer at the a ITO/MAPbl 3 or $\mathbf{b}$ ITO/F4TCNQ-doped $\mathrm{MAPbl}_{3}$ interface
ITO is thus facilitated, resulting in a reduced series resistance to increase $\mathrm{FF}^{35}$.

The diode Eq. (1) predicts that $R_{\mathrm{s}}$ variation has no influence on device $V_{\mathrm{oc}}$, while in our case, the F4TCNQ doping increased device $V_{\text {oc }}$ from 1.00 to $1.10 \mathrm{~V}$. This discrepancy indicates there is another factor, in addition to $R_{\mathrm{s}}$, which leads to the $V_{\mathrm{oc}}$ enhancement. To find out the reason, a transient photovoltage spectroscopy (TPV) measurement was also carried out to understand the charge carrier recombination dynamics. As shown in Supplementary Fig. 10, the $\mathrm{MAPbI}_{3}$ :F4TCNQ-based PSC had a longer carrier lifetime $(0.69 \mu \mathrm{s})$ than the reference device based on neat $\mathrm{MAPbI}_{3}(0.30 \mu \mathrm{s})$ under one sun illumination. This result was consistent with the larger $V_{\text {oc }}$ for the device with doping. The observed carrier recombination lifetime enhancement could be a result of band-bending configuration change due to doping, though there may be other reasons that may also change the carrier recombination lifetime. For instance, for the device with undoped $\mathrm{MAPbI}_{3}$, the retarded hole extraction would increase the possibility of charge recombination, because they cannot be effectively extracted out.

\section{Discussion}

We demonstrated how molecular doping influences the series resistance and interfacial charge transfer within HTL-free PSC devices with or without F4TCNQ incorporated in the perovskite layer. In the absence of HTL, both the electrons and holes can transport to the ITO/perovskite interface and recombine there, leading to serious interfacial charge recombination and thus inferior charge collection ${ }^{36}$. In addition, the mismatched work functions between perovskite and ITO would induce an undesirable energy barrier that leads to inferior hole transfer. These situations are all very detrimental to device performance. Molecular doping of perovskite by F4TCNQ to some extent induces favorable interfacial band bending, which allows efficient transport of holes at ITO/perovskite interface (Fig. 4b) ${ }^{35}$.

Choosing an appropriate p-type dopant with proper molecular size and selected surface hydrophility with ITO substrate is promising to further improve the ITO/perovskite interfacial affinity. Owing to the larger molecular size of F4TCNQ than the interstitial sites in perovskite structure, the F4TCNQ concentrated at the grain boundary regions. The dopant assisted with charge dissociation and thus resulting in more balanced transfer of electrons and holes to their respective electrodes, which decreased the possibility of the charge carrier recombination. We found the charge trap density within $\mathrm{MAPbI}_{3}: \mathrm{F} 4 \mathrm{TCNQ}$-based PSC was even slightly smaller than its pristine $\mathrm{MAPbI}_{3}$ counterpart (Supplementary Fig. 11). It has been demonstrated that the trap sites within the organic semiconductors can be reduced due to partial filling by doping, typically at very low doping levels ${ }^{37}$.

In summary, an additive-assisted strategy for p-type molecular doping of solution-bladed perovskite films was demonstrated. F4TCNQ-doped $\mathrm{MAPbI}_{3}$ films show increased electrical conductivity, especially at grain boundary regions, and increased charge carrier concentrations. The incorporation of F4TCNQ in perovskite layers could modify the ITO/perovskite interface with reduced series resistance, which could be attributed to the favorable interfacial band bending for facilitated hole transfer and extraction from perovskite to ITO. The simple but effective approach enabled the scalable fabrication of HTL-free PSC devices with a simplified device geometry. Molecular doping of perovskite film by F4TCNQ led to the considerable enhancement of photovoltaic performance from 11.0 to $20.2 \%$. The $\mathrm{MAPbI}_{3}$ : F4TCNQ-based device exhibited a stabilized PCE that exceeded $20.0 \%$ and negligible hysteresis. This effective doping strategy 
eliminates the HTL preparation step, thus simplifying the PSC fabrication process and reducing costs. Extending the application of this doping technique to a broader range of semiconducting materials will definitely benefit the construction of other high performance, printed optoelectronic devices.

\section{Methods}

Materials preparation. Lead iodide $\left(\mathrm{PbI}_{2}, 99.9985 \%\right)$ was purchased from Alfa Aesar. Methylammonium iodide (MAI) was purchased from Dyesol. $\mathrm{MACl}(98 \%)$, 2,3,5,6-tetrafluoro-7,7,8,8-tetracyanoquinodimethane (F4TCNQ, 97\%), N,Ndimethylformamide (DMF, anhydrous, 99.8\%), DMSO (99.9\%), acetonitrile (anhydrous, 99.8\%) and CBZ (anhydrous, 99.8\%) were purchased from SigmaAldrich. All chemicals were used as received without further purification.

Device fabrication. The ITO glass substrates were ultrasonically washed with deionized water, acetone, and isopropanol for $30 \mathrm{~min}$ successively. After drying, the cleaned ITO glass was treated by UV-ozone (UVO) for 15 min then used immediately for device fabrication. To form a perovskite precursor $(1 \mathrm{M})$, an equimolar ratio of $\mathrm{PbI}_{2}$ and MAI was dissolved in DMF with different amounts of MHP (0.075 wt $\%$ to $0.300 \mathrm{wt} \%)$, F4TCNQ $(0.01 \mathrm{wt} \%$ to $0.05 \mathrm{wt} \%)$, and $0.5 \mathrm{wt} \% \mathrm{MACl}$ (for chlorine-containing precursor) added as required. Specifically, to implement solution-processed molecular doping of the perovskite photoactive layer, F4TCNQ was dissolved in DMF separately, and then added to the solution of as-prepared $\mathrm{MAPbI}_{3}$ precursor solution. Typically, MHP/DMF additive was used to increase grain size, improve perovskite film crystallinity, and surface coverage. The bladed coating of perovskite films was conducted in an $\mathrm{N}_{2}$-purged glovebox (below 1.0 ppm $\mathrm{O}_{2}$ and $\left.\mathrm{H}_{2} \mathrm{O}\right)$. The perovskite precursor solution $(\sim 5$ to $10 \mu \mathrm{L})$ was dripped onto the ITO glass on a hot plate set at $150^{\circ} \mathrm{C}$, then swiped linearly by a glass blade at a speed of $0.75 \mathrm{~cm} \mathrm{~s}^{-1}$. The as-prepared perovskite films were then annealed at $100^{\circ} \mathrm{C}$ for $30 \mathrm{~min}$. For solvent annealing, the perovskite films were annealed under a vapor of mixed DMSO/CBZ solvent $(1: 1 \mathrm{v} / \mathrm{v})$ at $100^{\circ} \mathrm{C}$ for $30 \mathrm{~min}$ according to a literature method ${ }^{31}$. Finally, $\mathrm{C}_{60}(20 \mathrm{~nm}$, Nano-C), bathocuproine (BCP, $8 \mathrm{~nm}$, Sigma-Aldrich), and $\mathrm{Cu}$ electrode $(80 \mathrm{~nm})$ were sequentially deposited on the perovskite layer by thermal evaporation to form a complete PSC device.

Device characterization. SEM images were obtained with a Quanta 200 FEG environmental SEM. X-ray diffraction (XRD) patterns were acquired by a Bruker D8 Discover Diffractometer with Cu Ka radiation (1.5406 ̊). PL spectrum was measured with a Horiba iHR320 Imaging Spectrometer at room temperature. A $532 \mathrm{~nm}$ green laser (Laserglow Technologies) with an intensity of $100 \mathrm{~mW} \mathrm{~cm}^{-2}$ was used as the excitation source. TRPL was obtained using the DeltaPro with a pulsed laser source at $406 \mathrm{~nm}$ (Horiba NanoLED 402-LH; pulse width below 200 ps, $20 \mathrm{pJ}$ per pulse, $\sim 1 \mathrm{~mm}^{2}$ spot size), and the signal was recorded using time corrected TCSPC. c-AFM measurements were performed on an Asylum Research MFP-3D AFM using Pt-coated Si conductive probes (PPP-EFM, Nanosensors). The conductivity of perovskite films was measured by a two-electrode method on glass substrates. The $J-V$ characteristics of the cells (voltage scanning rate $0.1 \mathrm{~V} \mathrm{~s}^{-1}$ ) and the steady photocurrent under maximum power output bias $(0.93 \mathrm{~V})$ were recorded with a Keithley 2400 source-meter under simulated AM 1.5 $\mathrm{G}$ irradiation by a Xenon lamp (Oriel 67005), which was calibrated by a silicon diode equipped with a Schott visible color KG5 glass filter. EQE was measured with a Newport QE measurement kit. The $J-V$ and EQE measurements were conducted under ambient air conditions without encapsulation. A non-reflective shadow mask was used to define a $0.08 \mathrm{~cm}^{2}$ active area of the PSCs. The series resistance $\left(R_{\mathrm{s}}\right)$ is derived from the slope of the $J-V$ curve at the open-circuit voltage point. TPV decay was measured under 1 sun illumination and recorded by a $1 \mathrm{GHz}$ Agilent digital oscilloscope.

Data availability. The data that support the findings of this study are available from the corresponding author on reasonable request.

Received: 23 November 2017 Accepted: 27 March 2018

Published online: 24 April 2018

\section{References}

1. Kojima, A., Teshima, K., Shirai, Y. \& Miyasaka, T. Organometal halide perovskites as visible-light sensitizers for photovoltaic cells. J. Am. Chem. Soc. 131, 6050-6051 (2009).

2. Correa-Baena, J.-P. et al. The rapid evolution of highly efficient perovskite solar cells. Energy Environ. Sci. 10, 710-727 (2017).

3. Li, X. et al. A vacuum flash-assisted solution process for high-efficiency largearea perovskite solar cells. Science 353, 58-62 (2016).
4. Yang, W. S. et al. Iodide management in formamidinium-lead-halide-based perovskite layers for efficient solar cells. Science 356, 1376-1379 (2017).

5. Zheng, X. et al. Defect passivation in hybrid perovskite solar cells using quaternary ammonium halide anions and cations. Nat. Energy 2, 17102 (2017).

6. Bi, D. et al. Efficient luminescent solar cells based on tailored mixed-cation perovskites. Sci. Adv. 2, e1501170 (2016).

7. Kim, J. H., Williams, S. T., Cho, N., Chueh, C.-C. \& Jen, A. K. Y. Enhanced environmental stability of planar heterojunction perovskite solar cells based on blade-coating. Adv. Energy Mater. 5, 1401229 (2015).

8. Deng, Y., Dong, Q., Bi, C., Yuan, Y. \& Huang, J. Air-stable, efficient mixedcation perovskite solar cells with $\mathrm{Cu}$ electrode by scalable fabrication of active layer. Adv. Energy Mater. 6, 1600372 (2016).

9. Deng, Y. et al. Scalable fabrication of efficient organolead trihalide perovskite solar cells with doctor-bladed active layers. Energy Environ. Sci. 8, 1544-1550 (2015).

10. Yang, M. et al. Perovskite ink with wide processing window for scalable high-efficiency solar cells. Nat. Energy 2, 17038 (2017).

11. Barrows, A. T. et al. Efficient planar heterojunction mixed-halide perovskite solar cells deposited via spray-deposition. Energy Environ. Sci. 7, 2944-2950 (2014).

12. Hwang, K. et al. Toward large scale roll-to-roll production of fully printed perovskite solar cells. Adv. Mater. 27, 1241-1247 (2015).

13. He, M. et al. Meniscus-assisted solution printing of large-grained perovskite films for high-efficiency solar cells. Nat. Commun. 8, 16045 (2017).

14. Chen, H., Wei, Z., Zheng, X. \& Yang, S. A scalable electrodeposition route to the low-cost, versatile and controllable fabrication of perovskite solar cells. Nano Energy 15, 216-226 (2015).

15. Deng, Y., Wang, Q., Yuan, Y. \& Huang, J. Vividly colorful hybrid perovskite solar cells by doctor-blade coating with perovskite photonic nanostructures. Mater. Horiz. 2, 578-583 (2015).

16. Tang, S. et al. Composition engineering in doctor-blading of perovskite solar cells. Adv. Energy Mater. 7, 1700302 (2017).

17. $\mathrm{Bi}, \mathrm{C}$. et al. Non-wetting surface-driven high-aspect-ratio crystalline grain growth for efficient hybrid perovskite solar cells. Nat. Commun. 6, 7747 (2015).

18. Ye, S. et al. CuSCN-based inverted planar perovskite solar cell with an average PCE of 15.6\%. Nano. Lett. 15, 3723-3728 (2015).

19. Ameen, S. et al. Perovskite solar cells: influence of hole transporting materials on power conversion efficiency. ChemSusChem 9, 10-27 (2016).

20. Wu, W.-Q. et al. Integrated planar and bulk dual heterojunctions capable of efficient electron and hole extraction for perovskite solar cells with $17 \%$ efficiency. Nano Energy 32, 187-194 (2017).

21. Etgar, L. et al. Mesoscopic $\mathrm{CH}_{3} \mathrm{NH}_{3} \mathrm{PbI}_{3} / \mathrm{TiO}_{2}$ heterojunction solar cells. J. Am. Chem. Soc. 134, 17396-17399 (2012)

22. $\mathrm{Li}, \mathrm{Y}$. et al. Hole-conductor-free planar perovskite solar cells with $16.0 \%$ efficiency. J. Mater. Chem. A 3, 18389-18394 (2015).

23. Tsai, K.-W., Chueh, C.-C., Williams, S. T., Wen, T.-C. \& Jen, A. K. Y. High performance hole-transporting layer-free conventional perovskite/fullerene heterojunction thin-film solar cells. J. Mater. Chem. A 3, 9128-9132 (2015).

24. Ye, S. et al. A Strategy to simplify the preparation process of perovskite solar cells by co-deposition of a hole-conductor and a perovskite layer. Adv. Mater. 28, 9648-9654 (2016).

25. Ye, S. et al. A breakthrough efficiency of $19.9 \%$ obtained in inverted perovskite solar cells by using an efficient trap statepassivator $\mathrm{Cu}$ (thiourea)I. J. Am. Chem. Soc. 139, 7504-7512 (2017).

26. Zhang, Y. et al. Molecular doping enhances photoconductivity in polymer bulk heterojunction solar cells. Adv. Mater. 25, 7038-7044 (2013).

27. Kirchartz, T. et al. Sensitivity of the Mott-Schottky analysis in organic solar cells. J. Phys. Chem. C. 116, 7672-7680 (2012).

28. Shao, Y. et al. Grain boundary dominated ion migration in polycrystalline organic-inorganic halide perovskite films. Energy Environ. Sci. 9, 1752-1759 (2016).

29. Duong, D. T. et al. Direct observation of doping sites in temperaturecontrolled, p-doped $\mathrm{P}_{3} \mathrm{HT}$ thin films by conducting atomic force microscopy. Adv. Mater. 26, 6069-6073 (2014)

30. Xiao, Z. et al. Unraveling the hidden function of a stabilizer in a precursor in improving hybrid perovskite film morphology for high efficiency solar cells. Energy Environ. Sci. 9, 867-872 (2016).

31. Wu, W.-Q., Chen, D., Huang, F., Cheng, Y.-B. \& Caruso, R. A. Optimizing semiconductor thin films with smooth surfaces and well-interconnected networks for high-performance perovskite solar cells. J. Mater. Chem. A 4, 12463-12470 (2016).

32. Wang, Q., Bi, C. \& Huang, J. Doped hole transport layer for efficiency enhancement in planar heterojunction organolead trihalide perovskite solar cells. Nano Energy 15, 275-280 (2015).

33. Schulz, P. et al. Electronic level alignment in inverted organometal perovskite solar cells. Adv. Mater. Interfaces 2, 1400532 (2015). 
34. Wang, Q. et al. Qualifying composition dependent $\mathrm{p}$ and $\mathrm{n}$ self-doping in $\mathrm{CH}_{3} \mathrm{NH}_{3} \mathrm{PbI}_{3}$. Appl. Phys. Lett. 105, 163508 (2014).

35. Lange, I. et al. Band bending in conjugated polymer layers. Phys. Rev. Lett. 106, 216402 (2011).

36. Cui, P. et al. Highly efficient electron-selective layer free perovskite solar cells by constructing effective $\mathrm{p}-\mathrm{n}$ heterojunction. Sol. RRL 1, 1600027 (2017).

37. Jacobs, I. E. \& Moulé, A. J. Controlling molecular doping in organic semiconductors. Adv. Mater. 29, 1703063 (2017).

\section{Acknowledgements}

We thank the financial support from Office of Naval Research under awards N00014-151-2713 and N00014-17-1-2619, and National Science Foundation under awards DMR1505535 and DMR-1420645.

\section{Author contributions}

J.H. conceived the idea. W.-Q.W. conducted most of device fabrication and device measurement. S.T. and Y.D. conducted the film morphology and XRD measurement. Y.F. carried out the PL, carrier lifetime, and trap density study. Q.W., Y.S., H.L., Y.L., T.L., Z.Y., and A.G. contributed to the conductivity and surface work function study. J.H. and W.-Q.W. wrote the manuscript. All authors discussed the results and commented on the manuscript.

\section{Additional information}

Supplementary Information accompanies this paper at https://doi.org/10.1038/s41467018-04028-8
Competing interests: The authors declare no competing interests.

Reprints and permission information is available online at http://npg.nature.com/ reprintsandpermissions/

Publisher's note: Springer Nature remains neutral with regard to jurisdictional claims in published maps and institutional affiliations.

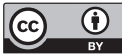

Open Access This article is licensed under a Creative Commons Attribution 4.0 International License, which permits use, sharing, adaptation, distribution and reproduction in any medium or format, as long as you give appropriate credit to the original author(s) and the source, provide a link to the Creative Commons license, and indicate if changes were made. The images or other third party material in this article are included in the article's Creative Commons license, unless indicated otherwise in a credit line to the material. If material is not included in the article's Creative Commons license and your intended use is not permitted by statutory regulation or exceeds the permitted use, you will need to obtain permission directly from the copyright holder. To view a copy of this license, visit http://creativecommons.org/ licenses/by/4.0/

(C) The Author(s) 2018 